\title{
Desempenho motor e dificuldades de aprendizagem em escolares com idades entre 7 e 10 anos
}

\author{
Motor performance and learning difficulties in schoolchildren aged 7 to 10 \\ years old
}

\section{J. Silva, T.S. Beltrame}

RESUMO

O objetivo geral deste estudo foi avaliar o desempenho motor de crianças com e sem indicativos de dificuldades de aprendizagem. Participaram do estudo 406 escolares com idades entre 7 e 10 anos, sendo 231 (56.9\%) meninas e 175 (43.1\%) meninos, estudantes de uma escola pública municipal de São José/SC/Brasil. O indicativo de dificuldades de aprendizagem foi verificado por meio do Teste de Desempenho Escolar (TDE), enquanto o desempenho motor e o indicativo de dificuldades motoras com a Bateria para a Avaliação do Movimento da Criança (MABC). Meninos sem dificuldades de aprendizagem tiveram melhor desempenho na maior parte das habilidades avaliadas, além de haver associação entre o indicativo de problemas motores com as dificuldades de aprendizagem em escrita, matemática e leitura. Por outro lado, estudantes do sexo feminino com e sem indicativo de dificuldades de aprendizagem não se diferenciaram quanto às habilidades motoras avaliadas, havendo somente associação entre o indicativo de dificuldades motoras e as dificuldades de leitura. Com base nas diferenças identificadas entre meninos e meninas, os resultados chamam a atenção sobre a necessidade de que pesquisas futuras nesta área considerem a variável sexo como um diferencial nessa relação. Palavras-chave: desempenho motor, habilidades motoras, dificuldades de aprendizagem, dificuldades motoras, escolares

ABSTRACT

The general objective of this study was to evaluate the motor performance of children with and without learning difficulty indicatives. Took part in the study 406 students aged 7 to 10 years old, being 231 girls $(56.9 \%)$ and 175 (43.1\%) boys enrolled in a municipal public school in São José, Santa Catarina, Brazil. The indicative of learning difficulties was verified through the TDE, while motor performance was evaluated with the MABC. Boys without learning difficulties had better performance in the majority of the abilities evaluated, beyond an association between the indicative of motor problems with learning difficulties towards writing, arithmetic, reading, and in general. On the other hand, female students of the sample with and without any indicative of learning difficulties did not differentiate themselves as to motor abilities evaluated, with an association merely between the indicative of motor problems and reading problems. Based on the differences identified between girls and boys, results call attention to the need for future research in this area, considering gender as a differential variable in this relationship.

Keywords: motor performance, motor abilities, learning difficulties, motor difficulties, schoolchildren

Submetido: 21.02.2010 | Aceite: 31.07.2010

Juliana da Silva. Doutoranda em Ciências do Movimento Humano pela Universidade do Estado de Santa Catarina - UDESC; Laboratório de Distúrbios da Aprendizagem e do Desenvolvimento - LADADE, Brasil.

Thaís Silva Beltrame. Laboratório de Distúrbios da Aprendizagem e do Desenvolvimento - LADADE; Universidade do Estado de Santa Catarina - UDESC, Brasil.

Endereço para correspondência: Thaís Silva Beltrame, Centro de Ciências da Saúde e do Esporte (CEFID/UDESC), Laboratório de Distúrbios da Aprendizagem e do Desenvolvimento, Rua Pascoal Simone, 358 - Coqueiros, CEP: 88080-350 Florianópolis - SC, Brasil.

E-mail: thais.beltrame@udesc.br 
As causas mais frequentes apontadas pela literatura para o baixo desempenho escolar são as dificuldades de aprendizagem. Tais dificuldades, de acordo com Rebelo (1993; cit. por Capellini, Tonelotto, \& Ciasca, 2004, p. 80) “...podem ser entendidas como barreiras encontradas por alunos durante o período de escolarização, referentes à captação ou assimilação dos conteúdos", devendo ser diferenciadas das variações normais na realização acadêmica e das dificuldades escolares devido à falta de oportunidades, má qualidade de ensino, dentre outros (APA, 1995). As dificuldades de aprendizagem podem estar associadas a outros fatores que podem dificultar o desempenho em tarefas escolares, tais como: condição socioeconômica, nutrição inadequada, localização da escola, pouca motivação dos professores, problemas motores e emocionais, baixo senso de auto-eficácia, entre outros (Capellini et al., 2004; Cunha, 1990; Fávero \& Calsa, 2004; Medeiros, Loureiro, Linhares, \& Maturano, 2003; Stevanato, Loureiro, Linhares, \& Maturano, 2003). Há que se considerar ainda que os escolares nessa condição se encontram em posição de risco psicossocial, o que os coloca em desvantagem educacional e social (Santos \& Graminha, 2005). Quanto à prevalência das dificuldades de aprendizagem, estudos realizados com populações internacionais sugerem que pelo menos $5 \%$ dos escolares apresentam estes problemas (Smith \& Strick, 2001).

Nesta fase da vida, o desempenho motor encontra-se em constante evolução, partindo de movimentos mais simples e realizados de maneira individualizada, para uma série de combinações, que virão a ser utilizadas em atividades de lazer, esportivas e principalmente cotidianas, concomitante aos processos da aprendizagem escolar e do amadurecimento das principais habilidades motoras (Gallahue \& Ozmun, 2005). O equilíbrio se torna eficiente, os padrões motores básicos estão mais refinados e adaptados às diferenças estruturais, há uma melhora na coordenação motora, bem como ocorre um aumento na proficiência de habilidades manipulativas (Gallahue \& Ozmun, 2005).

Para Cunha (1990), há uma possível relação entre o desenvolvimento motor e a aprendizagem escolar, sendo que esta relação se apresenta mais acentuadas nos primeiros anos do ensino fundamental, onde a criança está sendo alfabetizada e que coincide com o período em que costumam aparecer os primeiros sinais das dificuldades de aprendizagem (Fávero \& Calsa, 2004). Porém, algumas crianças ao atingirem a idade escolar não possuem o esperado domínio de suas habilidades motoras básicas e passam a ter um desempenho aquém do esperado, sendo que em alguns casos estes problemas são significativamente graves (Ferreira, Nascimento, Apolinário, \& Freudenheim, 2006).

A prevalência de dificuldades motoras na população de crianças em idade escolar varia de $6 \%$ a $8 \%$ (APA, 1995), sendo que um grande número destas apresenta também dificuldades específicas na aprendizagem, como dislexia e distúrbios específicos da linguagem (Kaplan, Wilson, Dewey, \& Crawford, 1998; Ramus, Pidgeon, \& Frith, 2003; Visser, 2003). Estimase que $30 \%$ a $50 \%$ das crianças que apresentam dificuldades motoras, também possuem dificuldades de aprendizagem associadas, o que varia de acordo com os padrões utilizados para a avaliação das dificuldades (O 'Hare \& Khalid, 2002; Ramus et al., 2003; Smits-Engelsman, Niemeijer, \& Galen, 2001). As dificuldades na escrita e leitura são as mais comumente associadas aos problemas motores, em comparação à dificuldade de aprendizagem da matemática (Smits-Engelsman et al., 2001).

No início da escolarização é possível identificar crianças com dificuldades na escrita, atendo-se principalmente ao seu comportamento motor (Fávero \& Calsa, 2004). O desenvolvimento da motricidade fina possui extrema relação com a boa aquisição da escrita, sendo verificado problemas na aprendizagem da escrita em grande parte das crianças que possuem dificuldades motoras finas (Souza \& Sisto, 2001). Desta forma, pode-se dizer que 
problemas referentes ao planejamento e a coordenação motora, podem, se não causar, acentuar as dificuldades na aquisição da escrita (Smits-Engelsman et al., 2001).

Apesar de os estudos evidenciarem as relações entre as dificuldades motoras e a aprendizagem escolar, poucas pesquisas brasileiras têm se direcionado a este tema. Acredita-se haver a necessidade de estudos mais aprofundados, referentes à incidência e prevalência de dificuldades motoras e da aprendizagem em crianças brasileiras, uma vez que esse fenômeno ainda é desconhecido da população em geral, o que faz com que as crianças que apresentam tais dificuldades não recebam um tratamento adequado. Desta forma, acredita-se que o aperfeiçoamento das habilidades motoras realizado juntamente com o reforço das disciplinas escolares, pode proporcionar benefícios para diversas atividades diárias, sendo estes verificados na escola, nos esportes, em momentos de brincadeiras, entre outros (Kadesjo \& Gillberg, 1999; Missiuna, Rivard, \& Pollock, 2004; Polatajko \& Cantin, 2005; Smits-Engelsman et al., 2001).

Outro fator importante está relacionado ao enfoque destinado às pesquisas que envolvem as dificuldades de aprendizagem, principalmente no Brasil, onde a maior parte dos estudos se direciona a outros aspectos, tais como: avaliação das dificuldades específicas da aprendizagem e funções neurológicas ligadas à aprendizagem; aspectos psicológicos; influência do sistema educacional; problemas comportamentais, socioculturais e socioeconômicos (Bandeira, Rocha, Freitas, Prete, \& Prete, 2006; Capovilla, Gütschow, \& Capovilla, 2004; Correia, 2004; Cia \& Barham, 2008; Dell'Aglio \& Hutz, 2004; Salles \& Parente, 2006; Schirmer, Fontoura, \& Nunes, 2004).

Tendo em vista esta lacuna existente na literatura brasileira sobre o tema até então apresentado, o objetivo geral deste estudo foi avaliar o desempenho motor de crianças com e sem indicativos de dificuldades de aprendizagem. De forma mais específica, objetivou-se: a) verificar a associação entre sexo e indicativo de dificuldades de aprendizagem e motoras; b) comparar o desempenho motor de crianças do sexo masculino e feminino com e sem indicativo de dificuldades de aprendizagem; e, c) verificar a associação entre o indicativo de dificuldades de aprendizagem e motoras de crianças do sexo masculino e feminino.

\section{MÉTODO}

\section{Amostra}

Participaram do estudo 231 (56.9\%) meninas e 175 (43.1\%) meninos, totalizando 406 escolares com idade entre 7 e 10 anos. A idade média foi de $8.75(D P=1.00)$ anos, sendo semelhante para as meninas $(M=8.74$, $D P=1.02)$ e meninos $(M=8.77, D P=.99)$.

Os alunos cursavam o ensino fundamental em turmas de segundas-séries $(n=145)$, terceiras-séries $(n=137)$ e quartas-séries $(n=$ 124), nos períodos matutino $(n=123)$ e vespertino $(n=283)$, em uma escola de educação básica localizada na região central do município de São José/SC, Brasil. Eram estudantes pertencentes às classes socioeconômicas baixa e média, sendo que $65 \%$ apresentaram renda familiar mensal de até três salários mínimos (atualmente o salário mínimo brasileiro é de $\mathrm{R} \$ 510.00$, aproximadamente US\$283.00).

A escolha da instituição se deu de forma intencional, por se tratar de uma das maiores escolas da região e possuir um número significativo de escolares que se enquadravam nos critérios da pesquisa.

\section{Instrumentos}

Teste de Desempenho Escolar (TDE; Stein, 1994)

O TDE foi confeccionado para a avaliação de escolares brasileiros de $1^{\mathrm{a}}$ a $6^{\mathrm{a}}$ séries do ensino fundamental (Stein, 1994). O instrumento tem sido amplamente utilizado em estudos realizados em diferentes regiões do país (Brancalhone, Fogo, \& Williams, 2004; Capellini et al., 2004; Cia \& Barham, 2008; D'Avila-Bacarji, Matunano, \& Elias, 2005; 
Medeiros, Loureiro, Linhares, \& Maturano, 2000) mostrando-se adequado para a avaliação da aprendizagem em escolares brasileiros. Com base no resultado do TDE é possível indicar dificuldades em escrita, matemática, leitura, ou geral. O teste é único e aplicável para todas as séries; porém para cada uma existe um padrão diferente de comparação, sendo que, quanto maior a pontuação, melhor é o desempenho no teste. Há três classificações para o desempenho escolar: superior, médio e inferior, sendo esta última um indicativo de dificuldade de aprendizagem, que pode ser específica (dificuldade de escrita, leitura ou aritmética), ou geral.

\section{Bateria para a Avaliação do Movimento da} Criança (MABC; Henderson \& Sugden, 1992)

A MABC tem sido utilizada para identificação de dificuldades motoras em crianças de diversos países com idades entre 4 e 12 anos, como: Reino Unido (Henderson \& Sugden, 1992), Austrália (Livesey, Coleman, \& Piek, 2007; Mon-Williams, Wann, \& Pascal, 1994), Singapura (Wright \& Sugden, 1996), Suécia (Kadesjo \& Gillberg, 1999), Grécia (Kourtessis et al., 2008) e Brasil (França, 2008; Souza, Ferreira, Catuzzo, \& Corrêa, 2007). O teste possui quatro conjuntos de tarefas, cada um apropriado a uma faixa etária específica. Para a realização desta pesquisa foram utilizados os testes para a faixa etária dois (7 e 8 anos) e três (9 e 10 anos). Este instrumento avalia as destrezas manuais, habilidades com bola e equilíbrio das crianças. Os escores das destrezas manuais e do equilíbrio variam de zero a quinze pontos, enquanto as habilidades com bola variam de zero a dez pontos. Quanto melhor o desempenho da criança nos testes, menor é a sua pontuação. Ao término das avaliações estes valores são somados, resultando no valor total da MABC, que pode variar de zero a 40 pontos, sendo esta a pior pontuação possível no teste.

O resultado final da MABC (MABC total) está pautado na soma dessas três habilidades motoras (que podem também ser analisadas em separado). Este resultado é comparado à tabela de percentis presente no protocolo da MABC. Com base neste procedimento são possíveis três classificações: problema motor definido (valores abaixo do $5^{\circ}$ percentil), problema motor limítrofe (valores entre o $5^{\circ} \mathrm{e}$ o $15^{\circ}$ percentil) e habilidades motoras normais (valores acima do $15^{\circ}$ percentil). A classificação problema motor definido é considerada um indicativo de dificuldades motoras.

\section{Procedimentos}

Para a realização do estudo, foi realizada a submissão deste ao Comitê de Ética em Pesquisa em Seres Humanos - CEP da Universidade do Estado de Santa Catarina UDESC, o qual aprovou todos os procedimentos pré-estabelecidos (número de referência 28/2008).

A coleta dos dados foi realizada em uma escola da rede pública de ensino do município de São José/SC, Brasil, que atende escolares em todos os seguimentos do ensino fundamental. Aos escolares com idade entre 7 e 10 anos foram entregues os termos de consentimento livre e esclarecido, para serem preenchidos e assinados pelos pais e/ou responsáveis, para autorização das crianças no estudo. Foram autorizados 406 escolares.

O primeiro teste aplicado foi o TDE, a fim de avaliar o desempenho escolar dos alunos e identificar aqueles com indicativo de dificuldades de aprendizagem, isto é, que tenham apresentado resultado inferior no teste. Em seguida, os escolares passaram pela avaliação motora, por meio da MABC. Os testes foram realizados em ambiente isolado, sem influências externas.

A coleta dos dados foi realizada no período compreendido entre maio e novembro de 2008, respeitando o espaço de recesso escolar, ocorrido no mês de julho do mesmo ano.

\section{Análise Estatística}

A análise dos dados foi realizada por meio de estatística descritiva e inferencial. Quanto à 
estatística descritiva, utilizou-se a média, mediana, desvio padrão, frequência e percentual, bem como a verificação da distribuição dos dados por meio do teste Kolmogorov-Smirnov, o qual demonstrou não haver nenhuma variável com distribuição normal. Desta forma optou-se pela utilização apenas de testes estatísticos não paramétricos na análise inferencial dos dados.

Utilizou-se o teste $U$ de Mann-Whitney para comparação dos resultados referentes às habilidades motoras (destrezas manuais, habilidades com bola e equilíbrio) de meninos e meninas e crianças com e sem indicativo de dificuldades de aprendizagem. A associação entre os dados categóricos, como desempenho motor e dificuldades de aprendizagem, ou ainda entre estes e os sexos, foi realizada por meio do teste Qui-Quadrado $\left(\chi^{2}\right)$, com coeficiente $\mathrm{V}$ de Cramer para verificação da força das associações. Em todas as análises estatísticas foi estabelecido um $\alpha$ de .05 .

\section{RESULTADOS}

Quanto às dificuldades de aprendizagem específicas, houve um maior percentual de escolares com dificuldades em escrita $(n=$ 178), seguido de dificuldades em matemática $(n=146)$ e leitura $(n=101)$. Cento e vinte sete alunos apresentaram dificuldades gerais. Nenhuma das dificuldades esteve associada ao sexo (ver tabela 1). A maior parte dos escolares apresentou habilidades motoras normais $(n=$ 293, 72.2\%), seguido de problemas motores limítrofes $(n=65,16.7 \%)$ e problemas motores definidos ( $n=45,11.1 \%)$. Não se verificou associação entre os sexos e o indicativo de dificuldades motoras $\left(\chi^{2}{ }_{(2)}=\right.$ 2.045, $p=.360)$, umas vez que os percentuais de meninos e meninas com problemas motores definidos foram semelhantes, assim como nas demais classificações da avaliação motora (ver figura 1).

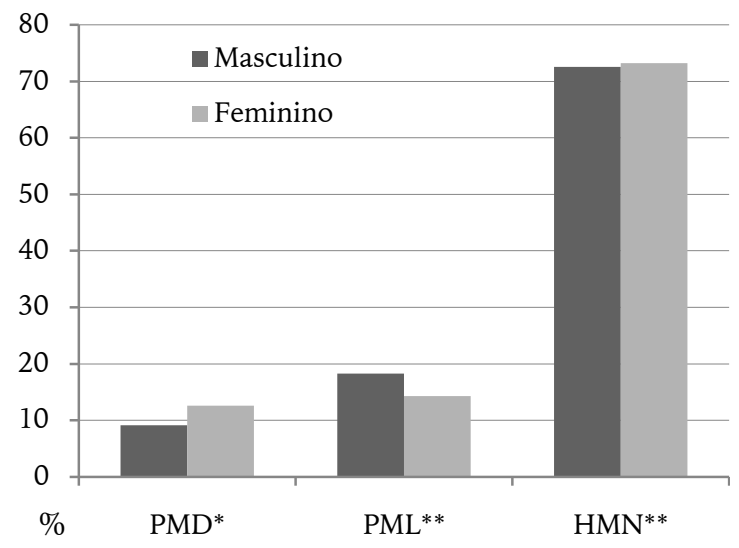

Figura 1. Percentual e frequência de meninos e meninas para as diferentes classificações da MABC

(Nota: * Problema Motor Definido, ** Problema Motor Limítrofe e ${ }^{* * *}$ Habilidades Motoras Normais)

Ao compararmos o desempenho motor dos meninos com e sem dificuldades de aprendizagem (ver tabela 2), verificou-se um melhor desempenho nas destrezas manuais, equilíbrio e MABC total $(p<.05)$ para os escolares sem dificuldades, exceto para a dificuldade de matemática, em relação ao equilíbrio, e dificuldade de leitura, em relação às destrezas manuais. As habilidades com bola não se diferenciaram entre os meninos com e sem os diferentes tipos de dificuldades de aprendizagem.

Tabela 1

Percentual e frequência de crianças do sexo masculino e feminino com dificuldades específicas e gerais de aprendizagem

\begin{tabular}{ccccc}
\hline & $\begin{array}{c}\text { Dificuldades em } \\
\text { Escrita } \\
\%(n)\end{array}$ & $\begin{array}{c}\text { Dificuldades em } \\
\text { Matemática } \\
\%(n)\end{array}$ & $\begin{array}{c}\text { Dificuldades em } \\
\text { Leitura } \\
\%(n)\end{array}$ & $\begin{array}{c}\text { Dificuldades } \\
\text { Gerais } \\
\%(n)\end{array}$ \\
\hline Meninos & $44.0 \%(77)$ & $34.3 \%(60)$ & $25.7 \%(45)$ & $36.0 \%(63)$ \\
Meninas & $43.7 \%(101)$ & $37.2 \%(86)$ & $24.2 \%(56)$ & $27.7 \%(64)$ \\
$\chi^{2}(p)^{*}$ & $.003(.956)$ & $.375(.540)$ & $.115(.734)$ & $3.187(.074)$ \\
\hline
\end{tabular}

${ }^{*}$ Teste Qui-Quadrado para associação entre dificuldades e sexo 
Tabela 2

Médias, medianas e desvios padrão das habilidades motoras dos escolares em função da existência ou não das dificuldades de aprendizagem dos meninos

\begin{tabular}{|c|c|c|c|c|c|c|c|c|c|c|c|c|c|}
\hline & & \multicolumn{3}{|c|}{$\begin{array}{l}\text { Destrezas } \\
\text { Manuais }\end{array}$} & \multicolumn{3}{|c|}{$\begin{array}{c}\text { Habilidades } \\
\text { com Bola }\end{array}$} & \multicolumn{3}{|c|}{ Equilíbrio } & \multicolumn{3}{|c|}{ MABC Total } \\
\hline & & $M$ & $M d n$ & $D P$ & $M$ & $M d n$ & $D P$ & $M$ & $M d n$ & $D P$ & $M$ & $M d n$ & $D P$ \\
\hline \multirow{3}{*}{$\begin{array}{l}\text { Dificuldades } \\
\text { em Escrita }\end{array}$} & Sim & 3.94 & 3.50 & 3.16 & 1.01 & .00 & 1.86 & 3.13 & 3.00 & 2.65 & 8.14 & 8.00 & 5.02 \\
\hline & Não & 2.67 & 2.00 & 2.72 & .80 & .00 & 1.64 & 2.37 & 1.50 & 2.31 & 5.88 & 4.75 & 4.47 \\
\hline & $p^{*}$ & & .008 & & & .324 & & & .048 & & & .002 & \\
\hline \multirow{3}{*}{$\begin{array}{l}\text { Dificuldades } \\
\text { em Matemática }\end{array}$} & Sim & 4.28 & 4.25 & 3.14 & 1.22 & .00 & 2.14 & 2.96 & 3.00 & 2.32 & 8.46 & 8.50 & 4.88 \\
\hline & Não & 2.68 & 2.00 & 2.76 & .72 & .00 & 1.47 & 2.57 & 2.00 & 2.27 & 6.05 & 5.00 & 4.62 \\
\hline & $p^{*}$ & & .001 & & & .263 & & & .146 & & & .001 & \\
\hline \multirow{3}{*}{$\begin{array}{l}\text { Dificuldades } \\
\text { em Leitura }\end{array}$} & Sim & 3.77 & 3.00 & 3.13 & 1.02 & .00 & 2.11 & 3.34 & 3.50 & 2.60 & 8.20 & 8.50 & 5.02 \\
\hline & Não & 3.04 & 2.50 & 2.92 & .84 & .00 & 1.59 & 2.48 & 2.00 & 2.41 & 6.42 & 5.50 & 4.70 \\
\hline & $p^{*}$ & & .164 & & & .783 & & & .042 & & & .031 & \\
\hline \multirow{3}{*}{$\begin{array}{c}\text { Dificuldades } \\
\text { Gerais }\end{array}$} & Sim & 3.87 & 3.50 & 3.11 & 1.04 & .00 & 1.95 & 3.33 & 3.00 & 2.77 & 8.32 & 8.50 & 5.20 \\
\hline & Não & 2.86 & 2.00 & 2.86 & .80 & .00 & 1.61 & 2.35 & 1.75 & 2.25 & 6.06 & 5.00 & 4.44 \\
\hline & $p^{*}$ & & .020 & & & .732 & & & .017 & & & .005 & \\
\hline
\end{tabular}

* Teste U de Mann-Whitney para análise das diferenças nas habilidades motoras dos escolares com e sem as dificuldades de aprendizagem; Nota: Os valores de média e mediana mais elevados indicam pior desempenho na MABC

Tabela 3

Médias, medianas e desvios padrão das habilidades motoras dos escolares em função da existência ou não das dificuldades de aprendizagem das meninas

\begin{tabular}{|c|c|c|c|c|c|c|c|c|c|c|c|c|c|}
\hline & & \multicolumn{3}{|c|}{$\begin{array}{l}\text { Destrezas } \\
\text { Manuais }\end{array}$} & \multicolumn{3}{|c|}{$\begin{array}{c}\text { Habilidades } \\
\text { com Bola }\end{array}$} & \multicolumn{3}{|c|}{ Equilíbrio } & \multicolumn{3}{|c|}{ MABC Total } \\
\hline & & $M$ & $M d n$ & $D P$ & $M$ & $M d n$ & $D P$ & $M$ & $M d n$ & $D P$ & $M$ & $M d n$ & $D P$ \\
\hline \multirow{3}{*}{$\begin{array}{c}\text { Dificuldades } \\
\text { em Escrita }\end{array}$} & Sim & 2.77 & 1.50 & 3.25 & 1.67 & 1.00 & 2.00 & 2.09 & 1.50 & 2.28 & 6.61 & 5.00 & 5.23 \\
\hline & Não & 2.84 & 2.00 & 2.75 & 1.77 & 1.00 & 2.07 & 2.22 & 1.50 & 2.42 & 6.88 & 6.50 & 4.83 \\
\hline & $p^{*}$ & & .317 & & & .609 & & & .616 & & & .467 & \\
\hline \multirow{3}{*}{$\begin{array}{l}\text { Dificuldades } \\
\text { em Matemática }\end{array}$} & Sim & 2.91 & 2.00 & 3.10 & 1.74 & 1.00 & 1.87 & 2.15 & 1.00 & 2.64 & 6.92 & 6.25 & 5.06 \\
\hline & Não & 2.75 & 1.50 & 2.90 & 1.72 & 1.00 & 2.14 & 2.17 & 1.50 & 2.18 & 6.67 & 6.00 & 4.98 \\
\hline & $p^{*}$ & & .676 & & & .524 & & & .473 & & & .760 & \\
\hline \multirow{3}{*}{$\begin{array}{c}\text { Dificuldades } \\
\text { em Leitura }\end{array}$} & Sim & 2.79 & 2.00 & 3.13 & 1.89 & 1.25 & 1.85 & 2.84 & 2.25 & 2.99 & 7.58 & 6.50 & 5.74 \\
\hline & Não & 2.31 & 1.50 & 2.98 & 1.67 & 1.00 & 2.10 & 1.95 & 1.50 & 2.08 & 6.50 & 6.00 & 4.73 \\
\hline & $p^{*}$ & & .645 & & & .202 & & & .070 & & & .315 & \\
\hline \multirow{3}{*}{$\begin{array}{c}\text { Dificuldades } \\
\text { Gerais }\end{array}$} & Sim & 2.91 & 2.00 & 3.37 & 1.66 & 1.00 & 1.79 & 2.25 & 1.75 & 2.49 & 6.85 & 5.00 & 5.57 \\
\hline & Não & 2.77 & 2.00 & 2.81 & 1.75 & 1.00 & 2.13 & 2.13 & 1.50 & 2.31 & 6.73 & 6.00 & 4.78 \\
\hline & $p^{*}$ & & .567 & & & .876 & & & .969 & & & .781 & \\
\hline
\end{tabular}

* Teste U de Mann-Whitney para análise das diferenças nas habilidades motoras dos escolares com e sem as dificuldades de aprendizagem; Nota: Os valores de média e mediana mais elevados indicam pior desempenho na MABC 
No caso das meninas (ver tabela 3), em nenhuma das habilidades motoras avaliadas houve diferença estatisticamente significativa entre as participantes com e sem indicativos de dificuldades de aprendizagem.

Entre os meninos (ver tabela 4), verificaram-se associações entre as dificuldades de aprendizagem e o indicativo de dificuldades motoras $(p<.05)$, com resultados inferiores para os escolares que apresentaram dificuldades de aprendizagem. Entre as meninas (ver tabela 4), houve associação apenas entre os problemas motores e as dificuldades de aprendizagem em leitura $(p<.05)$, sendo que as escolares com estas dificuldades apresentaram pior desempenho motor. As associações verificadas para ambos os sexos foram fracas, de acordo com o coeficiente $\mathrm{V}$.

\section{DISCUSSÃO}

O presente estudo pretendeu avaliar o desempenho motor de crianças com e sem indicativos de dificuldades de aprendizagem.

Não houve associação significativa entre sexo e indicativo de dificuldades de aprendizagem, contrariando outras pesquisas brasileiras (Meister et al., 2001; Santos \& Graminha, 2005) e internacionais (Rutter et al., 2004; Saigal, Hoult, Stoskopf, Rosenbaum, \& Streiner, 1998) que apontam maior proporção de meninos com dificuldades de aprendizagem em comparação às meninas. Embora não confirmado estatisticamente, ao se comparar os sexos o presente estudo mostra mais meninas com dificuldades de aritmética, enquanto os meninos apresentaram mais dificuldades em leitura, escrita e gerais. Essa

Tabela 4

Classificação da MABC em função da existência ou não das dificuldades de aprendizagem de meninos e meninas

\begin{tabular}{|c|c|c|c|c|c|c|}
\hline & & $\begin{array}{c}\text { Problema Motor } \\
\text { Definido* } \\
\%(n)\end{array}$ & $\begin{array}{c}\text { Problema Motor } \\
\text { Limítrofe } \\
\%(n)\end{array}$ & $\begin{array}{c}\text { Habilidades } \\
\text { Motoras Normais } \\
\%(n)\end{array}$ & $\begin{array}{l}\chi^{2} \\
(p)\end{array}$ & $\begin{array}{c}\text { V de } \\
\text { Cramer }\end{array}$ \\
\hline \multicolumn{7}{|c|}{ Meninos $(n=175)$} \\
\hline \multirow{2}{*}{$\begin{array}{l}\text { Dificuldades } \\
\text { em Escrita }\end{array}$} & Sim & $15.6 \%(12)$ & $19.5 \%(15)$ & $64.9 \%(50)$ & 7.222 & \multirow{2}{*}{.203} \\
\hline & Não & $4.1 \%(4)$ & $18.4 \%(18)$ & $77.6 \%(76)$ & $(.027)$ & \\
\hline \multirow{2}{*}{$\begin{array}{l}\text { Dificuldades } \\
\text { em Matemática }\end{array}$} & Sim & $18.3 \%(11)$ & $18.3 \%(11)$ & $63.3 \%(38)$ & 9.401 & \multirow{2}{*}{.232} \\
\hline & Não & $4.3 \%(5)$ & $19.1 \%(22)$ & $76.5 \%(88)$ & $(.009)$ & \\
\hline \multirow{2}{*}{$\begin{array}{l}\text { Dificuldades } \\
\text { em Leitura }\end{array}$} & Sim & $22.2 \%(10)$ & $15.6 \%(7)$ & $62.2 \%(28)$ & 13.926 & \multirow{2}{*}{.267} \\
\hline & Não & $4.6 \%(6)$ & $20.0 \%(26)$ & $75.4 \%(98)$ & $(.002)$ & \\
\hline \multirow{2}{*}{$\begin{array}{l}\text { Dificuldades } \\
\text { Gerais }\end{array}$} & Sim & $17.5 \%(11)$ & $15.9 \%(10)$ & $66.7 \%(42)$ & 8.302 & \multirow{2}{*}{.218} \\
\hline & Não & $4.5 \%(5)$ & $20.5 \%(23)$ & $75.0 \%(84)$ & $(.016)$ & \\
\hline \multicolumn{7}{|c|}{ Meninas $(n=231)$} \\
\hline \multirow{2}{*}{$\begin{array}{l}\text { Dificuldades } \\
\text { em Escrita }\end{array}$} & Sim & $14.9 \%(15)$ & $11.9 \%(12)$ & $73.3 \%(74)$ & \multirow{2}{*}{$\begin{array}{l}2.045 \\
(.360)\end{array}$} & \multirow{2}{*}{-} \\
\hline & Não & $10.8 \%(14)$ & $17.7 \%(23)$ & $71.5 \%(93)$ & & \\
\hline \multirow{2}{*}{$\begin{array}{l}\text { Dificuldades } \\
\text { em Matemática }\end{array}$} & Sim & $15.1 \%(13)$ & $12.8 \%(11)$ & $72.1 \%(62)$ & \multirow{2}{*}{$\begin{array}{l}1.221 \\
(.543)\end{array}$} & \multirow{2}{*}{ - } \\
\hline & Não & $11.0 \%(16)$ & $16.6 \%(24)$ & $72.4 \%(105)$ & & \\
\hline \multirow{2}{*}{$\begin{array}{l}\text { Dificuldades } \\
\text { em Leitura }\end{array}$} & $\operatorname{Sim}$ & $17.9 \%(10)$ & $23.2 \%(13)$ & $58.9 \%(33)$ & \multirow{2}{*}{$\begin{array}{l}6.654 \\
(.036)\end{array}$} & \multirow{2}{*}{.170} \\
\hline & Não & $10.9 \%(19)$ & $12.6 \%(22)$ & $76.6 \%(134)$ & & \\
\hline \multirow{2}{*}{$\begin{array}{l}\text { Dificuldades } \\
\text { Gerais }\end{array}$} & Sim & $17.2 \%(11)$ & $10.9 \%(7)$ & $71.9 \%(46)$ & \multirow{2}{*}{$\begin{array}{l}2.554 \\
(.279)\end{array}$} & \multirow{2}{*}{-} \\
\hline & Não & $10.8 \%(18)$ & $16.8 \%(28)$ & $75.5 \%(121)$ & & \\
\hline
\end{tabular}

* Indicativo de dificuldades motoras 
tendência vai ao encontro dos dados divulgados pelo Instituto Nacional de Estudos e Pesquisas Educacionais Anísio Teixeira (INEP, 2009) a respeito do desempenho escolar de estudantes brasileiros, onde se tem um melhor desempenho das meninas nas avaliações de língua portuguesa e dos meninos nas avaliações de matemática. Porém, o que chama a atenção é a evolução no desempenho acadêmico das meninas, que estão cada vez mais se sobressaindo nos testes de língua portuguesa e reduzindo a diferença para os meninos em relação à matemática (INEP, 2009).

Não houve também associação entre sexo e problemas motores, semelhante aos resultados encontrados na pesquisa de Cairney et al. (2005), onde não foram identificadas diferenças na proporção de meninos e meninas com esses problemas. Porém, estes resultados discordam da maior parte dos estudos, que apontam para uma maior prevalência no sexo masculino (Dewey \& Wilson, 2001; Kadesjo \& Gillberg, 1999; Wilson \& McKenzie, 1998; Wright \& Sugden, 1998). Há ainda pesquisas que apontam o contrário, um maior número de meninas com indicativo de problemas motores (França, 2008). Assim, acredita-se na impossibilidade de associá-los a um sexo específico, considerando as divergências existentes na literatura. Divergências estas, que podem ocorrer devido à escolha de diferentes instrumentos e parâmetros de normalidade, tipos de estudos, populações investigadas, entre outros fatores.

Há uma série de pesquisas que evidenciam a ligação entre as dificuldades de aprendizagem e a execução das habilidades motoras (Dewey, Kaplan, Crawford, \& Wilson, 2002; Fonseca, 1995; Jongmans, Smits-Engelsman, \& Schoemaker, 2003; Kourtessis et al., 2008; Moreira, Fonseca, \& Diniz, 2000; Ramus et al., 2003; Visser, 2003). Porém, esta ligação foi observada, no presente estudo, apenas no sexo masculino, onde se verificou um melhor desempenho dos meninos sem dificuldades de aprendizagem em quase todas as habilidades motoras avaliadas. Os resultados, vão ao encontro do apresentado por Kourtessis et al. (2008), em pesquisa realizada com escolares gregos de ambos os sexos, onde se verificou um melhor desempenho das crianças sem dificuldades de aprendizagem nas tarefas do MABC. Corroborando com estes resultados, Fávero e Calsa (2004) demonstraram que crianças com melhor desenvolvimento motor apresentam mais facilidade na aprendizagem da leitura e escrita. Segundo Smits-Engelsman et al. (2001), crianças com dificuldades específicas na escrita desenvolvem menos estratégias para desempenhar tarefas motoras finas, necessitando de maiores auxílios externos, ou feedbacks, provenientes de pais e professores.

Analisando as destrezas manuais dos grupos com e sem indicativos de dificuldades de escrita, verificou-se que somente para o sexo masculino houve diferenças significativas, onde os meninos sem indicativos de dificuldades de aprendizagem obtiveram um melhor desempenho nas atividades de destrezas manuais. De acordo com SmitsEngelsman et al. (2001), estes resultados se explicam pelo grande nível de precisão, regulação e coordenação motora fina necessários à escrita. Para Henderson e Sugden (1992), além de comprometer a escrita as dificuldades nas destrezas manuais podem, consequentemente, comprometer a atenção em sala de aula, o que fortalece ainda mais as dificuldades no contexto escolar.

Meninos com dificuldades de aprendizagem em escrita e leitura apresentaram piores resultados em seu equilíbrio, concordando com a literatura (Nicolson \& Fawcett, 2007; White et al., 2006). Porém, não se verificou nos estudos ora citados distinção entre os sexos nas análises, sendo as mesmas conclusões apontadas tanto para os meninos como para as meninas. Uma das principais hipóteses para explicar tal relação (problemas no equilíbrio com dificuldades de aprendizagem em escrita e leitura) é a existência de desordens cerebelares associadas a estes problemas específicos da 
aprendizagem (Eckert et al., 2003; Leornard et al., 2002; Reynolds \& Nicolson, 2007; Vicari et al., 2005) e que podem vir a prejudicar as funções do cerebelo responsáveis pela realização de tarefas motoras e regulação do equilíbrio e postura. Entretanto, White et al. (2006) contestam estas hipóteses baseados nos resultados de seus estudos, onde pelo menos um terço das crianças que apresentaram problemas graves de leitura e escrita não apresentaram problemas cerebelares; muitas crianças sem déficits de leitura e escrita apresentaram problemas cerebelares; e ainda, se houveram relações entre o mau funcionamento do cerebelo e problemas na leitura e escrita, estas são relativamente fracas, não sendo detectada nos testes estatísticos realizados. Moe-Nilssen, Helbostad, Talcott e Toennessen (2003) compararam o equilíbrio de grupos de crianças com e sem problemas de aprendizagem e também não verificaram diferenças entre os mesmos. Nestes estudos, os autores sugeriram maiores investigações, devido a este não ser um tema esgotado.

Ao contrário dos meninos, meninas com e sem dificuldades de aprendizagem não apresentaram diferenças significativas na execução das habilidades motoras. Embora não haja na literatura algo a respeito dessa diferença entre os sexos, é possível fazer algumas considerações a esse respeito. As dificuldades motoras apresentadas pelas meninas muitas vezes estão relacionadas à falta de estímulos (Junaid \& Fellowes, 2006), o que acontece com menor frequência entre os meninos. Assim, a probabilidade de meninos com mau desempenho terem realmente um problema de desenvolvimento motor é maior, e estes podem ser consequentemente mais propensos a apresentarem outros tipos de problemas. Por outro lado, meninas com mau desempenho por falta de estimulação seriam menos afetadas em outros segmentos, como na aprendizagem, por exemplo.

Separadamente, as dificuldades de aprendizagem e motoras podem causar sérios danos à vida de quem as possui, o que dizer então quando ambas afetam a mesma criança? Ao compararem as habilidades motoras de crianças com problemas motores em conjunto com dificuldades de aprendizagem e crianças que apresentavam apenas problemas motores, Jongmans et al. (2003) verificaram que os participantes que possuíam os problemas em conjunto tiveram pior desempenho nas destrezas manuais e no equilíbrio.

No presente estudo houve associação significativa entre o indicativo de dificuldades motoras e de aprendizagem, especialmente entre os meninos. Esse resultado é semelhante ao encontrado por Kourtessis et al. (2008), onde $64.8 \%$ dos participantes com dificuldades de aprendizagem apresentaram problemas motores definidos, contra $15.1 \%$ das crianças sem problemas de aprendizagem. Tais achados convergem para a ideia de que as dificuldades motoras podem estar ligadas a dificuldades em outros segmentos da vida da criança, destacando a importância dos profissionais da educação, especialmente os professores de Educação Física, estarem atentos a possíveis atrasos no desenvolvimento motor das crianças.

A realização deste estudo chama a atenção sobre a importância de apresentar os resultados separadamente para cada sexo, uma vez os meninos sem dificuldades de aprendizagem apresentaram um melhor desempenho motor em comparação com os meninos com indicativos de dificuldades de aprendizagem. Bem como as associações entre as dificuldades de aprendizagem e motoras estiveram mais presentes no sexo masculino, em detrimento da análise dos resultados do sexo feminino. Tendo em vista que a maior parte dos estudos identificados na literatura não controla o sexo em suas análises, os resultados chamam a atenção sobre a necessidade das pesquisas futuras considerarem esta variável como um diferencial nessa relação. Com base nos resultados aqui apresentados, bem como das demais pesquisas relatadas, acredita-se que este trabalho auxilia no conhecimento do campo científico das 
ciências do movimento humano e da educação, apresentando novas evidências a respeito do desenvolvimento motor e da aprendizagem escolar em crianças brasileiras.

\section{REFERÊNCIAS}

American Psychiatric Association - APA (1995). Manual diagnóstico e estatístico de transtornos mentais ( $4^{\mathrm{a}}$ ed.). Porto Alegre: Artes Médicas.

Bandeira, M., Rocha, S. S., Freitas, L. C., Prete, D. A. P., \& Prete, A. (2006). Competência acadêmica de crianças do ensino fundamental: Características sociodemográficas e relação com habilidades sociais. Interação em Psicologia, 10(1), 53-62.

Brancalhone, P. G., Fogo, J. C., \& Williams, L. C. A. (2004). Crianças expostas à violência conjugal: Avaliação do desempenho acadêmico. Psicologia: Teoria e Pesquisa, 20(2), 113-117.

Cairney, J., Hay, J., Faught, B., Wade, T., Corna, L., \& Flouris, A. (2005). Developmental coordination disorder, generalized self-efficacy toward physical activity, and participation in organized and free play activities. The Journal of Pediatrics, 147, 515-520.

Capellini, S. A., Tonelotto, J. M. F., \& Ciasca, S. M. (2004). Medidas de desempenho escolar: Avaliação formal e opinião dos professores. Estudos de Psicologia (Campinas), 21 (2),79-90.

Capovilla, A. G. S., Gütschow, C. R. D., \& Capovilla, F. C. (2004). Habilidades cognitivas que predizem competência de leitura e escrita. Psicologia: Teoria e Prática, 6(2), 13-26.

Cia, F., \& Barham, E. J. (2008). Estabelecendo relação entre autoconceito e desempenho acadêmico de crianças escolares. Psico, 39(1), 21-27.

Correia, L. M. (2004). Problematização das dificuldades de aprendizagem nas necessidades educativas especiais. Análise Psicológica, 22(2), 369-376.

Cunha, M. F. C. (1990). Desenvolvimento psicomotor e cognitivo: Influência na alfabetização de criança de baixa renda. Tese de Doutorado, Instituto de Psicologia da Universidade de São Paulo, São Paulo, Brasil.

D’Avila-Bacarji, K. M. G., Maturano, E. M., \& Elias, L. C. S. (2005). Suporte parental: Um estudo sobre crianças com queixas escolares. Psicologia em Estudo, 10(1), 107-115.
Dell'Aglio, D. D., \& Hutz, C. S. (2004). Depressão e desempenho escolar em crianças e adolescentes institucionalizados. Psicologia: Reflexão e Crítica, 17(13), 341-350.

Dewey, D., \& Wilson, B. N. (2001). Developmental coordination disorder: What is it? Physical and Occupational Therapy in Pediatrics, 20, 5-28.

Dewey, D., Kaplan, B. J., Crawford, S. G., \& Wilson, B. N. (2002). Developmental, coordination disorder: Associated problems in attention, learning, and psychosocial adjustment. Human Movement Science, 21 (5/6), 905-918.

Eckert, M. A., Leonard, C. M., Richards, T. L., Aylward, E. H., Thomson, J., \& Berninger, V. W. (2003). Anatomical correlates of dyslexia: Frontal and cerebellar findings. Brain, 126(2), 492-494.

Fávero, M. T. M., \& Calsa, G. C. (2004). Desenvolvimento psicomotor e aprendizagem da escrita. Comunicação apresentada no Seminário de Pesquisa do Programa de Pós-Graduação em Educação, Maringá, Brasil.

Ferreira, L. F., Nascimento, R. O., Apolinário, M. R., \& Freudenheim, A. M. (2006). Desordem da coordenação do desenvolvimento. Motriz, 12(3), 283-292.

Fonseca, V. (1995). Manual de observação psicomotora: Significação psiconeurológica dos fatores psicomotores. Porto Alegre: Artes Médicas.

França, C. (2008). Desordem coordenativa desenvolvimental em crianças de 7 e 8 anos de idade. Dissertação de Mestrado, Universidade do Estado de Santa Catarina, Florianópolis, SC, Brasil.

Gallahue, D. L., \& Ozmun, J. C. (2005). Compreendendo o desenvolvimento motor: Bebês, crianças, adolescentes e adultos ( $3^{\mathrm{a}}$ ed.). São Paulo: Phorte.

Henderson, S. E., \& Sugden, D. A. (1992). Movement assessment battery for children. London: Psychological Corporation.

Instituto Nacional de Estudos e Pesquisas Educacionais Anísio Teixeira - INEP (2009). Mulheres na educação básica. Consultado em 25 de Março de 2009, a partir de http://www. inep.gov.br/imprensa/noticias/censo/escolar/n ews09_04.htm

Jongmans, M. J., Smits-Engelsman, B. C. M., \& Schoemaker, M. M. (2003). Consequences of comorbidity of developmental coordination disorders and learning disabilities for severity and pattern of perceptual-motor dysfunction. Journal of Learning Disabilities, 36 (6), 528-537. 
Junaid, K., \& Fellowes, S. (2006). Gender differences in the attainment of motor skills on the movement assessment battery for children. Physical \& Occupational Therapy in Pediatrics, 26(1/2), 5-11.

Kadesjo, B., \& Gillberg, C. (1999). Developmental coordination disorder in Swedish 7-year-old children. Journal of the American Academy of Child E Adolescent Psychiatry, 38(7), 820-828.

Kaplan, B. J., Wilson, B. N., Dewey, D., \& Crawford, S. G. (1998). DCD may not be a discrete disorder. Human Movement Science, 17, 471-490.

Kourtessis, T., Thomaidou, E., Liveri-Kantere, A., Michalopoulou, M., Kourtessis, K., \& Kioumourtzoglou, E. (2008). Prevalence of developmental coordination disorder among Greek children with learning disabilities. European Psychomotricity Journal, 1 (2), 10-17.

Leonard, C. M., Lombardino, L. J., Walsh, K., Eckert, M. A., Mockler, J. L., Rowe, L. A., ... Bose, C. B. (2002). Anatomical risk factors that distinguish dyslexia from sli predict reading skill in normal children. Journal of Communication Disorders, 35, 501-531.

Livesey, D., Coleman, R., \& Piek, J. (2007). Performance on the movement assessment battery for children by Australian 3- to 5-yearold children. Child: Care, Health and Development, 33(6), 713-719.

Medeiros, P. C., Loureiro, S. R., Linhares, M. B. M., \& Maturano, E. M. (2000). A auto-eficácia e os aspectos comportamentais de crianças com dificuldade de aprendizagem. Psicologia: Reflexão e Crítica, 13(3), 327-336.

Medeiros, P. C., Loureiro, S. R., Linhares, M. B. M., \& Maturano, E. M. (2003). O senso de autoeficácia e o comportamento orientado para a aprendizagem em crianças com queixas de dificuldades de aprendizagem. Estudos de Psicologia (Natal), 8(1), 93-105.

Meister, E. K., Bruck, I., Antoniuk, S. A., Crippa, A. C. S., Muzzolon, S. C. B., Spessatto, A., \& Gregolin, R. (2001). Learning disabilities: Analysis of 69 children. Arquivos de Neuropsiquiatria, 59(2), 338-341.

Missiuna, C., Rivard, L., \& Pollock, N. (2004). They're bright but can't write: Developmental coordination disorder in school aged children. Teaching Exceptional Children Plus, 1(1). Consultado em 28 de Dezembro de 2009, a partir de http://escholarship.bc.edu/education/ tecplus/vol1/iss1/art3/
Moe-Nilssen, R., Helbostad, J. L., Talcott, J. B., \& Toennessen, F. E. (2003). Balance and gait in children with dyslexia. Experimental Brain Research, 150(2), 237-244.

Mon-Williams, M., Wann, J. P., \& Pascal, E. (1994). Ophthalmic factors in developmental coordination disorder. Adapted Physical Activity Quarterly, 11, 170-178.

Moreira, N. R., Fonseca, V., \& Diniz, A. (2000). Proeficiência motora em crianças normais e com dificuldades de aprendizagem: Estudo comparativo e correlacional com base no teste de proficiência motora de Bruininks-Oseretsky. Revista da Educação Física/UEM, 11 (1), 11-26.

Nicolson, R., \& Fawcett, A. J. (2006). Do cerebellar deficits underlie phonological problems in dyslexia? Developmental Science, 9(3), 259-262.

O'Hare, A., \& Khalid, S. (2002). The association of abnormal cerebellar function in children with developmental coordination disorder and reading difficulties. Dyslexia, 8(4), 234-248.

Polatajko, H. J., \& Cantin, N. (2005). Developmental coordination disorder (dyspraxia): An overview of the state of the art. Seminars in Pediatric Neurology, 2(4), 250-258.

Ramus, F., Pidgeon, E., \& Frith, U. (2003). The relationship between motor control and phonology in dyslexic children. Journal of Child Psychology and Psychiatry, 44, 712-722.

Reynolds, D., \& Nicolson, R. I. (2007). Follow-up of an exercise-based treatment for children with reading difficulties. Dyslexia, 13, 78-96.

Rutter, M., Caspi, A., Fergusson, D., Horwood, J. L., Goodman, R., Maughan, B., ... Carroll, J. (2004). Sex differences in developmental reading disability. The Journal of the American Medical Association, 291 (16), 2007-2012.

Saigal, S., Hoult, L. A., Stoskopf, B. L., Rosenbaum, P. L., \& Streiner, D. L. (1998). Role of gender and birthweight in the stability of psychometric scores and the incidence of specific learning disabilities (LD) among "normal" ELBW and term children. Pediatric Research, 43 (4), 228.

Salles, J. F., \& Parente, M. A. M. (2006). Funções neuropsicológicas em crianças com dificuldades de leitura e escrita. Psicologia: Teoria e Pesquisa, $22(2), 153-162$.

Santos, P. L., \& Graminha, S. S. V. (2005). Problemas emocionais e comportamentais associados ao baixo rendimento acadêmico. Estudos de Psicologia (Natal), 11 (1), 101-109. 
Schirmer, C. R., Fontoura, D. R., \& Nunes, M. L. (2004). Distúrbio da aquisição da linguagem e da aprendizagem. Jornal de Pediatria, 80(2), 85103.

Smith, C., \& Strick, L. (2001). Dificuldades de aprendizagem de A a Z: Um guia completo para pais e professores. Porto Alegre: Artmed.

Smits-Engelsman, B. C. M., Niemeijer, A. S., \& Galen, G. P. (2001). Fine motor deficiencies in children diagnosed as DCD based on poor grapho-motor ability. Human Movement Science, 20, 161-182.

Souza, A. R. M., \& Sisto, F. F. (2001). Dificuldade de aprendizagem em escrita, memória e contradições. Psicologia Escolar e Educacional, 5(2), 39-47.

Souza, C., Ferreira, L., Catuzzo, M. T., \& Corrêa, U, C. (2007). O teste ABC do movimento em crianças de ambientes diferentes. Revista Portuguesa de Ciência e Desporto, 7(1), 36-47.

Stein, L. M. (1994). Teste de desempenho escolar: TDE. São Paulo: Casa do Psicólogo.

Stevanato, I. S., Loureiro, S. R., Linhares, M. B. M., \& Maturano, E. M. (2003). Auto-conceito de crianças com dificuldades de aprendizagem e problemas de comportamento. Psicologia em Estudo, 8(1), 67-76.

Vicari, S., Finzi, A., Menghini, D., Marotta, L., Baldi, S., \& Petrosini, L. (2005). Do children with developmental dyslexia have an implicit learning deficit? Journal of Neurology, Neurosurgery \& Psychiatry, 76, 1392-1397.

Visser, J. (2003). Developmental coordination disorder: A review of research on subtypes and comorbidities. Human Movement Science, 22, 479 493.

White, S., Milne, E., Rosen, S., Hansen, P., Swettenham, J., Frith, U, \& Ramus, F. (2006). The role of sensorimotor impairments in dyslexia: A multiple case study of dyslexic children. Developmental Science, 9(3), 237-269.

Wilson, P. H., \& McKenzie, B. E. (1998). Information processing deficits associated with developmental coordination disorder: A metaanalyses of research findings. Journal of Child Psychology and Psychiatry, 39(6), 829-840.

Wright, H. C., \& Sugden, D. A. (1998). A school based intervention programme for children with developmental coordination disorder. Physical Education \& Sport Pedagogy, 3 (1), 35-50.

(cc) EY-NC Todo o conteúdo da revista Motricidade está licenciado sob a Creative Commons, excepto quando especificado em contrário e nos conteúdos retirados de outras fontes bibliográficas. 